\section{Cureus}

\title{
Indices of Regional Brain Atrophy: Formulae and Nomenclature
}

\author{
Manuel Menéndez González ${ }^{1}$, Oscar Arias-Carrión ${ }^{2}$ \\ 1. Neurology, Hospital Universitario Central de Asturias 2. Unidad de Trastornos del Movimiento y \\ Sueño, Hospital General Dr. Manuel Gea Gonzalez
}

$\square$ Corresponding author: Manuel Menéndez González, manuelmenendezgonzalez@gmail.com Disclosures can be found in Additional Information at the end of the article

\section{Abstract}

The pattern of brain atrophy helps to discriminate normal age-related changes from neurodegenerative diseases. Albeit indices of regional brain atrophy have proven to be a parameter useful in the early diagnosis and differential diagnosis of some neurodegenerative diseases, indices of absolute regional atrophy still have some important limitations. We propose using indices of relative atrophy for representing how the volume of a given region of interest (ROI) changes over time in comparison to changes in global brain measures over the same time.

A second problem in morphometric studies is terminology. There is a lack of systematization naming indices and the same measure can be named with different terms by different research groups or imaging softwares. This limits the understanding and discussion of studies.

In this technological report, we provide a general description on how to compute indices of absolute and relative regional brain atrophy and propose a standardized nomenclature.

Categories: Neurology, Psychiatry, Radiology

Keywords: brain atrophy, brain morphometry, mri, volumetry, planimetry, alzheimer's disease, parkinson's disease, clinical trials, neurodegenerative disease, aging

\section{Introduction}

Brain atrophies with age. Numerous cross-sectional and longitudinal imaging studies have found an inverse correlation between increasing age and decreasing brain volumes [1-7], and these findings are substantiated by postmortem data [8-9]. Grey matter (GM) volume loss appears to be a constant, linear function of age throughout adult life, whereas white matter (WM) volume loss seems to be delayed until middle adult life [10]. In any case, it is clear that brain volume decreases in healthy aging, and it can be observed in almost all brain areas over one year. Albeit changes are especially evident in temporal and prefrontal cortices, where the rate of annual decline is about $0.5 \%$ year, all subcortical and ventricular regions, except the caudate nucleus and the fourth ventricle, show changes visible in this period of time [11].

A better understanding of the brain aging process may help to discriminate normal age-related changes from neurodegenerative diseases. In neurodegenerative diseases, besides physiologic atrophy, patients develop a progressive focal atrophy that grows in extent and intensity with time. For instance, atrophy in Alzheimer's disease (AD) begins outside the hippocampus, with development of neurofibrillary tangles in the transentorhinal and entorhinal cortex, spreading subsequently to the subiculum and CA1 regions of the hippocampus and later on to other cortical areas [12-15]. A similar progression from focal to wide atrophy is seen in almost all 
neurodegenerative diseases. As a result, cortical atrophy and ventricular enlargement are present both in healthy aging and in all neurodegenerative diseases to some extent. However, the start point of regional atrophy, the rate of atrophy, and the pattern of atrophy progression varies between healthy aging and neurodegenerative disease and among neurodegenerative diseases.

Therefore, we sustain that we should use measures of relative regional atrophy comparing how a given structure changes in respect to global brain changes when studying neurodegenerative diseases. Moreover, as intracranial volume is an interindividual variant, all studies using absolute global or regional atrophy measures need to be normalized by intracranial volume. In the same line, studies found a highly significant and well-recognized effect of sex on volume, with men having larger brain volumes [2, 7]. This finding suggests that studies considering the effect of sex on cross-sectional volumes should also include a correction for head size to reduce this potential confounding effect. All these limitations are automatically solved when using relative rates of atrophy as each subject forms his or her own control.

Magnetic resonance imaging (MRI)-based measurements of the brain have been proposed as aids in the diagnosis of $\mathrm{AD}$ and other neurodegenerative diseases in clinical practice. Most studies assessing brain atrophy in neurodegenerative diseases have been done using volumetric techniques, although some others--specifically addressed to clinical practice--have used linear and planimetric techniques. Visual rating scales have also been developed, although these are not objective and, therefore, are not valid for assessing progression [16]. In any case, the rationale of using relative regional atrophy applies with all methodological approaches.

A second problem in morphometric studies is terminology, the lack of a homogeneous nomenclature for parameters and indices of atrophy. The same measure can be named with different names by different research groups or imaging software programs. For instance, a widely used measure, such as the yearly rate of Whole Brain Atrophy (yrWBA), is also known as yearly brain atrophy rate (yBAR) and annualised percent brain volume change (PBVC/y) [17]. This fact is a limitation for the comparison and discussion of results between groups.

The aim of this technological report is to provide a general description on how to compute indices of absolute and relative regional brain atrophy and propose a standardized nomenclature.

\section{Technical Report}

\section{Computing indices of absolute regional brain atrophy}

Computing the yearly rate of absolute regional brain atrophy is conceptually simple. First, we need the find out the volume of the target region (named Region of Interest, ROI) in the basal and follow-up MRI and compute the difference. Then all we need to do is to "annualize" the time lapsed between the two MRI studies (12/number of months). To do this, we just need to divide the number of months transcurred from basal to follow-up MRI studies. We recommend multiplying the result by 100 to avoid working with decimals.

Thus, the general formula for computing indices of absolute rate of atrophy is as follows:

$$
y r A-R O I=\frac{(R O I 1-R O I 2) \times 1200}{(\text { months between MRI studies })}
$$




\section{Cureus}

ROI2 the volume of the ROI in the follow-up MRI.

Indices can be computed for each hemisphere separately, or for both brain hemispheres together (taking the addition of volumes of ROI on both hemispheres). We will use cerebral hemispheres for telencephalic and diencephalic structures while we will use cerebellar hemispheres and ipsilateral brainstem for structures in the posterior fossa.

\section{Computing indices of relative regional brain atrophy}

In order to compute indices of relative atrophy, we need to find out the volume of the ROI and the volume of a brain structure used as a measure of reference (here named Ref). There are several potential Ref that can be used. The Ref is usually the whole brain volume, although it can also be other parameters, such as the cortical brain volume or ventricular volume (an inverse, indirect measure of brain atrophy). In this case, we use the lateral ventricles (I-II) with telencephalic ROI and the third ventricle (III) with diencephalic structures. When the ROI is in the posterior fossa, the Ref can be the volume of the parenchyma (brainstem plus cerebellar volumes) or the fourth ventricle (IV) as an inverse, indirect measure.

Thus, the general formula for computing indices of yearly rates of relative regional atrophy is as follows:

$y r R A-R O I(\operatorname{Ref})=\frac{(\text { ROI1 }- \text { ROI2 }) \times 1200}{(\text { Ref } 1-\text { Ref } 2) \times(\text { months between MRI studies })}$

where Ref is the short name of the measure of referece and ROI is the short name of the ROI.

ROI1 is the volume of the ROI in the first MRI and ROI2 the volume of the ROI in the second MRI.

Ref1 is the reference volume in the first MRI and Ref2 the reference volume in the second MRI. When using inverse measures of global atrophy such as ventricular volumes, we compute (Ref-Ref1) to avoid negative values.

Again, indices can be computed for each hemisphere separately, or for both hemispheres together. The ROI's ipsilateral reference must always be taken with paired reference structures.

\section{Nomenclature}

We propose a standardized, comprehensive terminology for naming the absolute and relative rates of atrophy of every ROI. The proposed nomenclature for the most frequently used ROI can be found in Table 1 .

ROI

Absolute Rate of Atrophy

Telencephalic ROI

Cortical Gray Matter
yrA-CGM
Relative Rate of Atrophy

yrRA-CGM(Ref) 


\section{Cureus}

Whole Brain Hemisphere

Brain Hemispheric-Cortical Gray Matter

Lobar Cortical Gray Matter (frontal, temporal, parietal, occipital and insular lobes)

Gyri (the name of any girus is suitable, following international nomina anatomica)

Forebrain Parenchyma

Hippocampus

Medial temporal lobe (hippocampus + parahippocamal gyrus)

Amygdala

Caudate

Putamen

Striatum (Caudate+Putamen)

Pallidum

Lenticularis nucleus (Putamen+Pallidum)

Diencephalic ROI

Thalamus

Hypothalamus

Subthalamic nucleus

ROI in the posterior fossa

Whole Cerebellum

Whole Cerebellar Hemisphere

Whole Cerebellar Cortical Gray Matter

Cerebellar Hemispheric Cortical Gray Matter

Midbrain (Mesencephalon)

Pons

Medulla

Olivary body

Substantia Nigra

Red Nucleus
yrA-WBHp
yrA-BHpCGM

yrRA-WBH(Ref)

yrA-BHCGM(Ref)

yrA-LCGM

yrA-LCGM(Ref)

yrA-"Gyrus name"

yrRA-"Gyrus

name"(Ref)

yrA-FP

yrRA-FP(Ref)

yrA-H

yrRA-H(Ref)

yrA-MTL

yrRA-MTL(Ref)

yrA-Amy

yrRA-Amy(Ref)

yrA-Cau

yrRA-Cau(Ref)

yrA-Pu

yrRA-Pu(Ref)

yrA-St

yrRA-St(Ref)

yrA-Pa

yrRA-Pa(Ref)

yrA-Len

yrRA-Len(Ref)

yrA-T

yrRA-T(Ref)

yrA-HypoT

yrRA-Gypot(Ref)

yrA-SubT

yrRA-SubT(Ref)

yrA-WCer

yrA-WCerHp

yrA-WCerCGM

yrA-CerHpCGM

yrA-MidB

yrA-Pons

yrA-Med

yrA-OB

yrA-Sn

yrA-Rn
yrRA-Cer(Ref)

yrRA-HCer(Ref)

yrRA-WCerCGM(Ref)

yrRA-CerHCGM(Ref)

yrRA-MidB(Ref)

yrRA-Pons(Ref)

yrRA-Med(Ref)

yrRA-OB(Ref)

yrRA-Sn(Ref)

yrRA-Rn(Ref)

TABLE 1: Nomenclature of Regions of Interest (ROI) and related indices of regional 


\section{Cureus}

\section{atrophy}

The most frequently used ROI with the corresponding nomenclature for absolute and relative rates of atrophy. The letters

"Ref" refer to the structure or paramenter used as measure of reference.

The first two letters denote the "temporal lapse", this way all indices start with "yr" for "yearly rate". Then we place the letter "A" if we are referring to an absolute rate of atrophy or the letters "RA" if we are referring to a relative rate of atrophy. Then a hyphen "-" separates the "yrA" or "yrRA" from the name of the ROI. Thus, the hyphen is followed by the initials of the anatomic structure used as ROI. When the ROI is a paired structure, the letter l (left) or r (right) must precede the initials of the structure if we are measuring side by side; otherwise, it will be understood that it is referred to both sides together. Finally, in rates of relative atrophy only, we add the initials of the referenced measure between parenthesis at the end "(Ref)", where (Ref) is the short name of the parameter or structure used as measure of reference (Table 2).

\section{Brain Structure}

\section{To be used with Telencephalic ROI}

Whole Brain

Brain Hemisphere

Cortical Gray Matter

Lateral Ventricles

To be used with Diencephalic ROI

Whole Brain

Third Ventricle

To be used with ROI in the posterior fossa

Whole Brain

Parenchyma in the posterior fossa

Cerebellar Hemisphere

Fourth Ventricle

\section{Initials or Symbol}

\section{WB}

$\mathrm{BHp}$

CGM

I-II

WB

III

\section{WB}

Ppf

CerHp

IV

\section{TABLE 2: Nomenclature of Structures Used as Reference}

Structures frequently used as reference in indices of relative rate of regional atrophy. Structures are arranged by the location of the ROI that will be used along.

Following this nomenclature, it is easy to read any index. For instance, the "yrA-Rn" reads "yearly rate of atrophy of the red nucleus" -and we understand it is a measure of absolute atrophy of both red nuclei- and the "yrRA-lT(WB)" reads "yearly rate of relative atrophy of the left 


\section{Discussion}

Regional brain atrophy has been proposed to be used in clinical practice for diagnosing neurodegenerative diseases and also as a surrogate marker of disease progression in clinical trials [16]. However, there is a lack of consensus on how to compute and nominate indices of regional brain atrophy.

Here, we describe a general approach for computing rates of absolute and relative regional atrophy--that may be extended to any ROI--at the time of proposing a standardized nomenclature. These indices are especially thought to be used with volumetric techniques, but they can also be applied to planimetric techniques where areas of ROI are used instead of volumes. Indeed, we have developed and validated some planimetric techniques, such as the yearly rate of Medial Temporal Lobe Atrophy (yrMTA) [18]--this should be named yrRA-MTL(III) in accordance to the terminology described here. The yrMTA has proven some usefulness in the diagnosis of $\mathrm{AD}$ (Poster presented at the Alzheimer's Association International Conference, Washington, 2015) and in correlating memory deficits in Parkinson's disease (PD) (Presented at the 18th Congress of Parkinson's Disease and Movement Disorders, Stockholm, 2014 and the 10th International Congress on Non-Motor Dysfunctions in Motor Dysfunctions in Parkinson's Disease, Nice, 2014). We have also described in detail some volumetric indices, such as the yearly rate of relative atrophy of the thalamic nuclei (yrRAT(I-II-III)) [19] that has proved helpful in the diagnosis of multiple sclerosis and in assessing the prognosis of patients with clinically isolated syndrome (Presented at the 31st ECTRIMS Congress, Barcelona, 2015).

If the methodological approach and nomenclature proposed here were adopted by all research groups working in brain morphometry, it would ease comparing and discussing results of studies addressing the rates of regional brain atrophy.

There is much work to do before any of these parameters are ready to be used with diagnostic purposes in routine clinical practice. Extensive research is needed in both retrospective and prospective studies. Retrospective studies are relatively easy to address, particularly for some conditions such as $\mathrm{AD}$ and $\mathrm{PD}$, where large databases of neuroimaging studies and clinical data are available to researchers worldwide. Those indices showing positive results in retrospective studies should be validated in prospective studies before they can be used in clinical practice and clinical trials.

\section{Conclusions}

There is a lack of consensus on how to compute and nominate indices of regional brain atrophy. Here, we provide a general description on how to compute indices of absolute and relative regional brain atrophy and propose a standardized nomenclature.

If this approach were universally adopted, it would allow a direct comparison of results from different research groups.

\section{Additional Information \\ Disclosures}

Human subjects: All authors have confirmed that this study did not involve human participants or tissue. Animal subjects: All authors have confirmed that this study did not involve animal subjects or tissue. Conflicts of interest: In compliance with the ICMJE uniform disclosure form, all authors declare the following: Payment/services info: All authors have 
declared that no financial support was received from any organization for the submitted work. Financial relationships: All authors have declared that they have no financial relationships at present or within the previous three years with any organizations that might have an interest in the submitted work. Other relationships: All authors have declared that there are no other relationships or activities that could appear to have influenced the submitted work.

\section{References}

1. Gur RC, Mozley PD, Resnick SM, Gottlieb GL, Kohn M, Zimmerman R, Herman G, Atlas S, Grossman R, Berretta D: Gender differences in age effect on brain atrophy measured by magnetic resonance imaging. Proc Natl Acad Sci U S A. 1991, 88:2845-2849.

10.1073/pnas.88.7.2845

2. Blatter DD, Bigler ED, Gale SD, Johnson SC, Anderson CV, Burnett BM, Parker N, Kurth S, Horn SD: Quantitative volumetric analysis of brain MR: normative database spanning 5 decades of life. AJNR Am J Neuroradiol. 1995, 16:241-251.

3. Mueller EA, Moore MM, Kerr DC, Sexton G, Camicioli RM, Howieson DB, Quinn JF, Kaye JA: Mueller EA, Moore MM, Kerr DC, Sexton G, Camicioli RM, Howieson DB, Quinn JF, Kaye JA . Neurology. 1998, 51:1555-1562. 10.1212/WNL.51.6.1555

4. Coffey CE, Wilkinson WE, Parashos IA, Soady SA, Sullivan RJ, Patterson LJ, Figiel GS, Webb MC, Spritzer CE, Djang WT: Quantitative cerebral anatomy of the aging human brain: a crosssectional study using magnetic resonance imaging. Neurology. 1992, 42:527-536. 10.1212/WNL.42.3.527

5. Murphy DG, DeCarli C, Schapiro MB, Rapoport SI, Horwitz B: Age-related differences in volumes of subcortical nuclei, brain matter, and cerebrospinal fluid in healthy men as measured with magnetic resonance imaging. Arch Neurol. 1992, 49:839-845. 10.1001/archneur.1992.00530320063013

6. Mu Q, Xie J, Wen Z, Weng Y, Shuyun Z: A quantitative MR study of the hippocampal formation, the amygdala, and the temporal horn of the lateral ventricle in healthy subjects 40 to 90 years of age. AJNR Am J Neuroradiol. 1999, 20:207-211.

7. Scahill RI, Frost C, Jenkins R, Whitwell JL, Rossor MN, Fox NC. A: Longitudinal Study of Brain Volume Changes in Normal Aging Using Serial Registered Magnetic Resonance Imaging. Arch Neurol. 2003, 989-994. 10.1001/archneur.60.7.989

8. Dekaban AS: Changes in brain weights during the span of human life: relation of brain weights to body heights and body weights. Ann Neurol. 1978, 4:345-356.

10.1002/ana.410040410

9. Ho KC, Roessmann U, Straumfjord JV, Monroe G: Analysis of brain weight, II: adult brain weight in relation to body height, weight, and surface area. Arch Pathol Lab Med. 1980, 104:640-645.

10. Ge Y, Grossman RI, Babb JS, Rabin ML, Mannon LJ, Kolson DL: Age-related total gray matter and white matter changes in normal adult brain. Part I: volumetric MR imaging analysis. Neuroradiol. 2002, 23:1327-33.

11. Fjell AM, Walhovd KB, Fennema-Notestine C, McEvoy LK, Hagler DJ, Holland D, Brewer JB, Dale AM: One-year brain atrophy evident in healthy aging . J Neurosci. 2009, 29:15223-31. 10.1523/JNEUROSCI.3252-09.2009

12. Jack CR Jr, Petersen RC, Xu YC, Waring SC, O'Brien PC, Tangalos EG, Smith GE, Ivnik RJ, Kokmen E: Medial temporal atrophy on MRI in normal aging and very mild Alzheimer's disease. Neurology. 1997, 49:786-794. 10.1212/WNL.49.3.786

13. Braak H: Braak E. On areas of transition between entorhinal allocortex and temporal isocortex in the human brain. Normal morphology and lamina-specific pathology in Alzheimer's disease. Acta Neuropathol. 1985:325-332. doi: 10.1007/BF00690836

14. Convit A, de Asis J, de Leon MJ, Tarshish CY, De Santi S, Rusinek H. Atrophy: of the medial occipitotemporal, inferior, and middle temporal gyri in non-demented elderly predict decline to Alzheimer's disease. Neurobiol. Aging, 2000:19-26. doi: 10.1016/S0197-4580(99)00107-4

15. Lim HK, Jung WS, Ahn KJ: et al. Relationships between hippocampal shape and cognitive performances in drug-naïve patients with Alzheimer's disease. Neurosci. Lett, 2012:124-129. doi: 10.1016/j.neulet.2012.03.072

16. Menéndez-González M, de Celis Alonso B, Salas-Pacheco J, Arias-Carrión O: Structural 


\section{Cureus}

neuroimaging of the medial temporal lobe in Alzheimer's disease clinical trials. J Alzheimers Dis. 2015, 48:in press.

17. Brainmarkers.com. (2015). Accessed: July 2, 2015:

http://brainatrophyindices.blogspot.com.es/p/nomenclature.html.

18. Conejo Bayón F, Maese J, Fernandez Oliveira A, Mesas T, Herrera de la Llave E, Alvarez Avellón T, Menéndez-González M: Feasibility of the Medial Temporal lobe Atrophy index (MTAi) and derived methods for measuring atrophy of the medial temporal lobe. Front Aging Neurosci. 2014, 6:305. 10.3389/fnagi.2014.00305

19. Menéndez-González M, Salas-Pacheco JM, Arias-Carrión O: The yearly rate of Relative Thalamic Atrophy (yrRTA): a simple 2D/3D method for estimating deep gray matter atrophy in Multiple Sclerosis. Front Aging Neurosci. 2014, 6:219. 10.3389/fnagi.2014.00219 